\title{
An entropic characterization of the flat metrics on the two torus
}

\author{
Patrick Bernard $^{1}$ • Clémence Labrousse ${ }^{1}$
}

Received: 30 May 2014 / Accepted: 24 June 2015 / Published online: 9 July 2015

(C) The Author(s) 2015. This article is published with open access at Springerlink.com

\begin{abstract}
The geodesic flow of the flat metric on a torus is minimizing the polynomial entropy among all geodesic flows on this torus. We prove here that this properties characterises the flat metric on the two torus.

Résumé Le flot géodésique des métriques plates sur un tore minimise l'entropie polynomiale parmi tous les flots géodésique sur ce tore. On montre ici que cette propriété caractérise les métriques plates en dimension deux.
\end{abstract}

Keywords Polynomail entropy $\cdot$ Rigidity $\cdot$ Weak KAM theory $\cdot$ Geodesic flow

Mathematics Subject Classification $\quad 53 \mathrm{D} 25 \cdot 53 \mathrm{C} 22 \cdot 37 \mathrm{~B} 40$

\section{Introduction}

There are several classes of hyperbolic manifolds on which the metrics with constant curvature are characterized by the fact that their geodesic flow is minimizing the topological entropy, see $[6,16]$ for example. The situation is different on tori. Flat metrics have zero entropy, but other metrics also have zero entropy, such as the tori of revolution. In order to characterize the flat metrics, it is therefore useful to consider a finer dynamical invariant of the geodesic flow, such as the polynomial entropy, introduced in [22].

The research leading to these results has received funding from the European Research Council under the European Union's Seventh Framework Programme (FP/2007-2013)/ERC Grant Agreement 307062.

Patrick Bernard

pbernard@dma.ens.fr

Clémence Labrousse

clemence.labrousse@ens.fr

1 DMA (UMR CNRS 8553), École Normale Supérieure, PSL, 45, rue d'Ulm, 75230 Paris Cedex 05, France 
Using the techniques of [22], it was proved in [17] that the polynomial entropy of a flat torus of dimension $d$ (in restriction to the sphere bundle) is equal to $d-1$, which is a lower bound for the polynomial entropy of all metrics on $\mathbb{T}^{d}$. It was also proved in [19] that the polynomial entropy of the revolution two torus is two, which is higher than the one of the flat two tori. This gives an indication that the polynomial entropy might be a sufficiently fine invariant to characterize the flat metrics. Our main result in the present paper is that this is indeed the case in dimension two. A partial result in that direction has been obtained in [18].

Theorem 1 If the polynomial entropy of a $C^{2}$ metric $g$ on $\mathbb{T}^{2}$ (in restriction to the sphere bundle) is smaller than two, then this entropy is equal to one and the torus $\left(\mathbb{T}^{2}, g\right)$ is isometric to a flat torus.

Theorem 1 immediately implies the following strong rigidity for flat metrics on $\mathbb{T}^{2}$ (see Corke and Kleiner [9]):

Corollary 1.1 Let $g$ be a flat metric on $\mathbb{T}^{2}$, and $g^{\prime}$ be another metric on $\mathbb{T}^{2}$. If the geodesics flows of $g$ and $g^{\prime}$ are $C^{0}$ conjugated, then $g$ and $g^{\prime}$ are isometric.

The detailed proof of Corollary 1.1 from Theorem 1 is given in Sect. 3.2 below. We will prove Theorem 1 using Mather-Fathi theory. The useful facts from this theory are recalled in Sect. 3, where a more general estimate on the polynomial entropy of Tonelli Hamiltonians is given, see Theorem 2. Theorem 1 is deduced from Theorem 2 using the Theorem of Hopf and its variants, see [15]. The definition of the polynomial entropy is recalled in Sect. 2, and the entropy estimates leading to the proof of Theorem 2 are detailed in Sect. 4. Once the dynamics has been well understood with the help of Mather-Fathy theory, these estimates are similar to those appearing in $[19,21,22]$.

\section{The polynomial entropy}

Consider a continuous map $f: X \rightarrow X$, where $(X, d)$ is a compact metric space. We construct new metrics $d_{n}^{f}$ on $X$ by setting

$$
d_{n}^{f}(x, y)=\max _{0 \leqslant k \leqslant n-1} d\left(f^{k}(x), f^{k}(y)\right) .
$$

These metrics are the dynamical metrics associated with $f$. Obviously, if $f$ is an isometry or is contracting, $d_{n}^{f}$ coincides with $d$ and in general $d_{n}^{f}$ is topologically equivalent to $d$. We denote by $G_{n}^{f}(\varepsilon)$ the minimal number of balls of radius $\varepsilon$ for the metric $d_{n}^{f}$ in a finite covering of $X$. The topological entropy of the $\operatorname{map} f$, defined as

$$
\mathrm{h}_{\text {top }}(f)=\lim _{\varepsilon \rightarrow 0} \limsup _{n \rightarrow \infty} \frac{\log G_{n}^{f}(\varepsilon)}{n}
$$

measures the exponential growth rate of $G_{n}^{f}$. In the present paper we will rather consider a polynomial measure of the growth rate introduced in [22]:

Definition 2.1 The polynomial entropy $\mathrm{h}_{\mathrm{pol}}(f)$ of $f$ is defined by

$$
\mathrm{h}_{\text {pol }}(f)=\lim _{\varepsilon \rightarrow 0} \limsup _{n \rightarrow \infty} \frac{\log G_{n}^{f}(\varepsilon)}{\log n} .
$$


We also consider sets that are $\varepsilon$-separated for the metrics $d_{n}^{f}$ (we will write $(n, \varepsilon)$ separated). Recall that a set $E$ is said to be $\varepsilon$-separated for a metric $d$ if for all $(x, y)$ in $E^{2}, d(x, y) \geq \varepsilon$. Denote by $S_{n}^{f}(\varepsilon)$ the maximal cardinal of a $(n, \varepsilon)$-separeted set contained in $X$.

Observing that $S_{n}^{f}(2 \varepsilon) \leqslant G_{n}^{f}(\varepsilon) \leqslant S_{n}^{f}(\varepsilon)$, we obtain

$$
\mathrm{h}_{\text {pol }}(f)=\lim _{\varepsilon \rightarrow 0} \limsup _{n \rightarrow \infty} \frac{\log S_{n}^{f}(\varepsilon)}{\log n} .
$$

Remark 2.1 If $\phi:=\left(\phi^{t}\right)_{t \in \mathbb{R}}$ is a continuous flow on $X$, for $t>0$ and $\varepsilon>0$, one can define in the same way the numbers $G_{t}^{\phi}(\varepsilon)$ and $S_{t}^{\phi}(\varepsilon)$. The polynomial entropy $\mathrm{h}_{\text {pol }}(\phi)$ of $\phi$ is defined as

$$
\mathrm{h}_{\text {pol }}(\phi)=\lim _{\varepsilon \rightarrow 0} \limsup _{t \rightarrow \infty} \frac{\log G_{t}^{\phi}(\varepsilon)}{\log t}=\lim _{\varepsilon \rightarrow 0} \limsup _{t \rightarrow \infty} \frac{\log S_{t}^{\phi}(\varepsilon)}{\log t} .
$$

One easily checks that if $\phi^{1}$ is the time-one map of $\phi, \mathrm{h}_{\mathrm{pol}}(\phi)=\mathrm{h}_{\mathrm{pol}}\left(\phi^{1}\right)$.

The following properties of the polynomial entropy are proved in [22].

Property 2.1 $1 . \mathrm{h}_{\mathrm{pol}}$ is a $C^{0}$ conjugacy invariant, and does not depend on the choice of topologically equivalent metrics on $X$.

2. If $A$ is an $f$-invariant subset of $X$, then $\mathrm{h}_{\mathrm{pol}}\left(f_{\left.\right|_{A}}\right) \leqslant \mathrm{h}_{\mathrm{pol}}(f)$.

3. For $m \in \mathbb{N}^{*}, \mathrm{~h}_{\mathrm{pol}}\left(f^{m}\right)=\mathrm{h}_{\mathrm{pol}}(f)$ and iff is invertible, $\mathrm{h}_{\mathrm{pol}}\left(f^{-m}\right)=\mathrm{h}_{\mathrm{pol}}(f)$.

We conclude this section with the following useful result which relates the polynomial entropy of a flow with that of a Poincaré map.

Proposition 2.1 Let $M$ be a smooth manifold, $d$ a distance on $M$ associated with a Riemannian metric, and $X$ a $C^{1}$ complete vector field on $M$ with flow $\phi=\left(\phi_{t}\right)_{t \in \mathbb{R}}$. Let $A$ a be compact $\phi$-invariant subset of $M$ and let $\Sigma$ be a $C^{1}$ codimension 1 embeddded submanifold of $X$ such that:

- for any $a \in A$, there exists $t>0$ such that $\phi^{t}(a) \in \Sigma$.

- for any $a \in A \cap \Sigma, X(a)$ is transverse to $\Sigma$.

Then the Poincaré return map $\varphi: A \cap \Sigma \rightarrow A \cap \Sigma$ is well defined, continuous and satisfies

$$
\mathrm{h}_{\mathrm{pol}}(\varphi) \leqslant \mathrm{h}_{\mathrm{pol}}\left(\phi_{\mid A}\right) .
$$

Proof Let $\tau: A \cap \Sigma \rightarrow \mathbb{R}_{+}^{*}: a \mapsto \tau_{a}$ be the first return time map of $\varphi$.

Since the function $\tau$ is continuous on the compact set $A \cap \Sigma$, we have $T:=\max \left\{\tau_{a} \mid a \in\right.$ $A \cap \Sigma\}<\infty$. Let $d_{\Sigma}$ be the distance induced by $d$ on $\Sigma$.

There exists $\tau^{*}>0$ and a neighborhood $V$ of $A \cap \Sigma$ in $\Sigma$ such that the map $\Phi$ :] $4 \tau^{*}, 4 \tau^{*}\left[\times V \cap \Sigma \rightarrow M:(t, a) \mapsto \phi^{t}(a)\right.$ is a $C^{1}$-diffeomorphism onto its image. Its inverse is thus locally Lipschitz, hence its restriction to the compact set $K:=\Phi\left(\left[-\tau^{*}, \tau^{*}\right] \times(A \cap \Sigma)\right)$ is Lipschitz. As a consequence, there exists $\delta>0$ such that for any $t, t^{\prime} \in\left[-\tau^{*}, \tau^{*}\right]$ and $a, a^{\prime} \in A \cap \Sigma$

$$
d\left(x, x^{\prime}\right) \geqslant \delta \max \left(\left|t-t^{\prime}\right|, d_{\Sigma}\left(a, a^{\prime}\right)\right) \text { if } x=\phi^{t}(a) \text { and } x^{\prime}=\phi^{t^{\prime}}\left(a^{\prime}\right) .
$$

Note that $\tau^{*}<\frac{1}{4} \min \left\{\tau_{a} \mid a \in A \cap \Sigma\right\}$. Since the compact sets $A \cap \Sigma$ and $A \backslash \Phi(]-\tau^{*}, \tau^{*}[\times V)$ are disjoint, the constant $\delta$ can be chosen such that

$$
d(a, x) \geqslant \delta \tau^{*} \text { for each } a \in \Sigma \cap A \text { and } x \in A \backslash K .
$$


Let $\tau_{x}^{k}$ be the successive return times of the point $x$, so that $\varphi^{k}(x)=\phi^{\tau_{x}^{k}}(x)$. Note that $\tau_{x}^{1}=\tau_{x}$, and $\tau_{x}^{k+1}=\tau_{x}^{k}+\tau_{\varphi^{k}(x)}$, hence $\tau_{x}^{k} \leqslant k T$ for all $x \in A \cap \Sigma$.

We will now prove that two points $x$ and $y$ of $A \cap \Sigma$ which are $(n, \varepsilon)$-separated by $\varphi$ are $(n T, \delta \varepsilon)$ separated by $\phi$ for $\varepsilon<\tau^{*}$. There exists $m \in\{0, \ldots, n\}$ such that $d_{\Sigma}\left(\varphi^{m}(x), \varphi^{m}(y)\right) \geqslant \varepsilon$. Let us assume for definiteness that $\tau_{x}^{m} \leqslant \tau_{y}^{m}$.

If $\phi^{\tau_{x}^{m}}(y) \in A \backslash K$, then $d\left(\phi^{\tau_{x}^{m}}(x), \phi^{\tau_{x}^{m}}(y)\right) \geqslant \delta \varepsilon$ by (2), hence $x$ and $y$ are $\left(\tau_{x}^{m}, \delta \varepsilon\right)$ separated by $\phi$.

If $\phi^{\tau_{x}^{m}}(y) \in K$, then there exists $m^{\prime} \leqslant m$ and $s \in\left[-\tau^{*}, \tau^{*}\right]$ such that $\phi^{\tau_{x}^{m}}(y)=$ $\phi^{s}\left(\varphi^{m^{\prime}}(y)\right)$.

If $m^{\prime}=m$, then $d\left(\phi^{\tau_{x}^{m}}(x), \phi^{\tau_{x}^{m}}(y)\right)=d\left(\varphi^{m}(x), \phi^{s}\left(\varphi^{m}(y)\right) \geqslant \delta \max (s, \varepsilon)\right.$, hence $x$ and $y$ are $\left(\tau_{x}^{m}, \delta \varepsilon\right)$-separated by $\phi$.

If $m^{\prime}<m$, since $\tau_{x}^{k+1}-\tau_{x}^{k}>2 \tau^{*}$ for $k \in\{1, \ldots, m-1\}$, there exists $k \in\{1, \ldots, m\}$ such that $\phi^{\tau_{x}^{k}}(y) \notin K$, otherwise $m^{\prime} \geqslant m$. Then $d\left(\phi^{\tau_{x}^{k}}(x), \phi^{\tau_{x}^{k}}(y)\right) \geqslant \delta \varepsilon$ by (2), hence the points $x$ and $y$ are $\left(\tau_{x}^{k}, \delta \varepsilon\right)$-separated by $\phi$.

We have proved that

$$
S_{n}^{\varphi}(\varepsilon) \leqslant S_{n T}^{\phi}(\delta \varepsilon)
$$

provided $\varepsilon<\tau^{*}$, which implies the inequality on $\mathrm{h}_{\text {pol }}$.

\section{Tonelli Hamiltonians}

\subsection{Some definitions from weak KAM theory}

We work on the $d$-dimensional torus $\mathbf{T}:=\mathbb{R}^{d} / \mathbb{Z}^{d}$, and will mostly consider the case $d=2$. A Tonelli Hamiltonian on $\mathbf{T}$ is a $C^{2}$ Hamiltonian function $H(q, p): \mathbf{T} \times \mathbb{R}^{d}\left(=T^{*} \mathbf{T}\right) \longrightarrow \mathbb{R}$ such that, for each $q \in \mathbf{T}$, the function $p \longmapsto H(q, p)$ is convex with positive definite Hessian and superlinear. The Hamiltonian vectorfield on $T^{*} \mathbf{T}$ is given by

$$
X_{H}(q, p)=\left(-\partial_{q} H(q, p), \partial_{p} H(q, p)\right) .
$$

It generates a complete flow $\varphi_{H}^{t}$ which preserves the function $H$.

To a Riemaniann metric $g_{x}\left(v, v^{\prime}\right)=\left\langle G(x) v, v^{\prime}\right\rangle$ where $G(x)$ is a $C^{2}$ field of positive definite symmetric matrices, we associate the Hamiltonian

$$
H(x, p)=\frac{1}{2}\left\langle G(x)^{-1} p, p\right\rangle .
$$

It is well-known that the Hamiltonian flow of $H$ is conjugated to the geodesic flow by the Legendre diffeomorphism $(x, v) \longmapsto(x, G(x) v)$. In other words, geodesics are the projections of Hamiltonian orbits.

Returning to the general case of a Tonelli Hamiltonian, the $\alpha$ function of Mather is defined on $H^{1}(\mathbf{T}, \mathbb{R})$ by

$$
\alpha(c):=\inf _{u \in C^{\infty}} \sup _{q} H(q, c+d u(q))=\min _{u \in C^{1,1}} \sup _{q} H(q, c+d u(q)),
$$

where the infimum and the minimum are taken respectively on the set of smooth functions on $\mathbf{T}$ and on the set of $C^{1}$ functions with Lipschitz differential. It was proved in [4] that the minimum exists on the set of $C^{1,1}$ functions, see also [11]. A $C^{1,1}$ function satisfying the inequality

$$
H(q, c+d u(q)) \leqslant \alpha(c)
$$


at each point $q \in M$ is called a $c$-critical subsolution (as we just recalled, such functions exist). There may exist several $c$-critical subsolutions. At least one of them, $w$, has the property that

$$
H(q, c+d w(q))=\alpha(c) \Rightarrow H(q, c+d u(q))=\alpha(c)
$$

for all critical subsolutions $u$. We define

$$
\mathcal{A}(c):=\{q \in \mathbf{T}, H(q, c+d w(q))=\alpha(c)\}=\cap_{u}\{q \in \mathbf{T}, H(q, c+d u(q))=\alpha(c)\},
$$

where the intersection is taken on all $c$-critical subsolutions $u$. This is a non-empty compact set, called the projected Aubry set. In view of the strict convexity of $H$ in $p$, the differential $d u(q)$ of a $c$-subsolution $u$ at a point $q \in \mathcal{A}(c)$ does not depend on the $c$-critical subsolution $u$. We define

$$
\left.\mathcal{A}^{*}(c):=\{(q, c+d w(q)), q \in \mathcal{A}(c)\}=\{(q, c+d u(q)), q \in \mathcal{A}(c))\right\}
$$

for each $c$-critical subsolution $u$. This set is called the Aubry set, it is invariant under the flow of $H$, compact, and not empty. It is moreover contained in the graph of the Lipschitz closed form $c+d u$ for each $c$-critical subsolution $u$. A consequence of the invariance of $\mathcal{A}^{*}(c)$ is that the projected Aubry $\mathcal{A}(c)$ set is invariant under the vectorfield

$$
\chi(q):=\partial_{p} H(q, c+d u(q))
$$

on $\mathbf{T}$ for each $c$-critical subsolution $u$. The special $c$-critical subsolution $w$ introduced above has the property that the strict inequality

$$
H(q, c+d w(q))<\alpha(c)
$$

holds on the complement of $\mathcal{A}(c)$. A $c$-critical subsolution having this property is said strict outside the Aubry set.

Mather measures at cohomology $c$ (also called $c$-minimizing measures) are defined as the invariant probability measures of the Hamiltonian flow supported on the Aubry set $\mathcal{A}^{*}(c)$. There is a bijection (given by the projection) between Mather measures at cohomology $c$ and $\chi$-invariant probability measures on $\mathcal{A}(c)$. We denote by $\mathcal{M}^{*}(c)$ the Mather set formed by the union of the supports of Mather measures at cohomology $c$. Its projection $\mathcal{M}(c)$, called the projected Mather set, is the union of supports of $\chi$-invariant probability measures on $\mathcal{A}(c)$. The Mather set $\mathcal{M}^{*}(c)$ is compact, non-empty, and invariant. The projected Mather set $\mathcal{M}(c)$ is compact, non-empty, and $\chi$-invariant. We define the rotation number of the Mather measure $\mu^{*}$ as

$$
h\left(\mu^{*}\right):=\int_{T^{*} \mathbf{T}} \partial_{p} H(q, p) d \mu^{*}(q, p)=\int_{\mathbf{T}} \chi(q) d \mu(q) \subset \mathbb{R}^{d}=H_{1}(\mathbf{T}, \mathbb{R}),
$$

where $\mu$ is the projection of $\mu^{*}$ on $\mathbf{T}$. So it is just the rotation number of $\mu$ seen as a $\chi$-invariant probability measure on $\mathbf{T}$.

There is an interesting relation between the function $c \longmapsto \alpha(c)$ and the rotation numbers of Mather measures, which was discovered by Mather [26]: The function $\alpha$ is convex and superlinear on $H^{1}(\mathbf{T}, \mathbb{R})$. Moreover, its subdifferential $\partial \alpha(c) \in H_{1}(\mathbf{T}, \mathbb{R})$ in the sense of convex analysis is precisely the set of rotation numbers of Mather measures at cohomology $c$.

It is well-known (and easy to prove using the convexity of $H$ ) that the inequality

$$
-\alpha(c) \leqslant \int_{T^{*} \mathbf{T}}(p-c) \cdot \partial_{p} H(q, p)-H(q, p) d \mu^{*}(q, p)
$$


holds for each compactly supported invariant probability measure $\mu^{*}$. Moreover, the invariant probability measures achieving equality are precisely the Mather measures at cohomology $c$. This explains the $c$-minimizing terminology. Our presentation however is not completely standard because we work on $T^{*} \mathbf{T}$ instead of $T \mathbf{T}$. If everything is sent back on $T \mathbf{T}$ using the Legendre transform, then the function $p \cdot \partial_{p} H(q, p)-H(q, p)$ is sent to the more familiar Lagrangian function.

Following Mather, we denote by $\beta(h): H_{1}(\mathbf{T}, \mathbb{R}) \longrightarrow \mathbb{R}$ the Legendre dual of $\alpha$. In the geodesic case, where $H$ is quadratic in the fibers, the functions $\alpha$ and $\beta$ are homogeneous of degree 2 . The function $\sqrt{\beta}$, which is homogeneous of degree one, is called the stable norm.

\subsection{Flat metrics and proof of the Corollary 1.1}

Let us discuss the case of flat metrics on $\mathbf{T}=\mathbb{T}^{d}$, which form an easy class of examples. A flat metric is isometric to a constant metric of the form $g_{q}(v, v)=\left\langle G v, v^{\prime}\right\rangle$ where $G$ (hence also $G^{-1}$ ) is a fixed positive definite symmetric matrix. The corresponding Hamiltonian function is

$$
H(q, p)=\frac{1}{2}\left\langle G^{-1} p, p\right\rangle .
$$

Proposition 3.1 If the Hamiltonian flows of two Hamiltonians $H(q, p)=\frac{1}{2}\left\langle G^{-1} p, p\right\rangle$ and $\tilde{H}(q, p)=\frac{1}{2}\left\langle\tilde{G}^{-1} p, p\right\rangle$ associated to constant metrics are topologically conjugated (in restriction to their energy levels $\{H=1\}$ and $\{\tilde{H}=1\})$, then there exists a matrix $A \in G l_{d}(\mathbb{Z})$ such that $G=A^{t} \tilde{G} A$. As a consequence, the corresponding metrics are isometric.

Proof Let us consider the quadratic functions $n(v)=\langle G v, v\rangle / 2$ and $\tilde{n}(v)=\langle\tilde{G} v, v\rangle / 2$ on $\mathbb{R}^{d}$ and let us denote $\Sigma$ and $\tilde{\Sigma}$ their unit spheres $\Sigma=\{n=1\}$. The map $(q, p) \longmapsto\left(q, G^{-1} p\right)$ conjugates the Hamiltonian flow of $H$ in restriction to the energy level $H=1$ to the flow of the equations $\dot{q}=v, \dot{v}=0$ on $\mathbf{T} \times \Sigma$. Since the flows of $H$ and $\tilde{H}$ are conjugated, there exists a homeomorphism $\varphi: \mathbf{T} \times \Sigma \longrightarrow \mathbf{T} \times \tilde{\Sigma}$ which conjugates the flows of $\dot{q}=v, \dot{v}=0$ on these manifolds.

For each non resonant $v \in \Sigma$, the torus $\mathbf{T} \times\{v\}$ is invariant, topologically transitive, and $d$-dimensional, hence it is sent by $\varphi$ to a torus with the same properties, which must be a torus of the form $\mathbf{T} \times\{w\}$, for some $w \in \tilde{\Sigma}$. By density of the non resonant vectors in $\Sigma$, we conclude that the map $\varphi$ is of the form $\varphi(q, v)=\left(\varphi_{1}(q, v), \phi(v)\right)$. Since $\varphi$ is a homeomorphism, so is $\phi: \Sigma \longrightarrow \tilde{\Sigma}$. In the case $d \geqslant 3$ the map $\varphi$ induces a homology map

$$
\varphi_{*}: \mathbb{Z}^{d}=H_{1}(\mathbf{T} \times \Sigma) \longrightarrow H_{1}(\mathbf{T} \times \tilde{\Sigma})=\mathbb{Z}^{d}
$$

which is given by a matrix $A \in G l_{d}(\mathbb{Z})$. In the case $d=2$, the action

$$
\varphi_{*}: \mathbb{Z}^{2} \times \mathbb{Z}=H_{1}(\mathbf{T} \times \Sigma) \longrightarrow H_{1}(\mathbf{T} \times \tilde{\Sigma})=\mathbb{Z}^{2} \times \mathbb{Z}
$$

is upper triangular by blocks, and the left upper bloc is a matrix $A \in G l_{2}(\mathbb{Z})$ which describes the action on homology of the restriction $\varphi_{\mid \mathbf{T} \times\{v\}}$ for each $v \in \Sigma$. For each $v \in \Sigma \cap\left(\mathbb{R}^{d}\right)$, each orbit of $\mathbf{T} \times\{v\}$ is periodic and gives rise to an oriented closed curve whose homology is the only indivisible element $h$ of $\mathbb{R}^{+} v \cap \mathbb{Z}^{d}$. Moreover, the minimal period of this orbit is the positive real number $T$ such that $T v=h$. The image by $\varphi$ of such a periodic orbit is a periodic orbit of minimal period $T$ and homology $\tilde{h}=A h$. Since this orbit belongs to $\mathbf{T} \times\{\phi(v)\}$, we conclude that $\tilde{h}=T \phi(v)$, hence that $\phi(v)=A v$. By continuity, this equality holds for each $v$, hence

$$
\tilde{\Sigma}=\phi(\Sigma)=A(\Sigma)
$$


This implies that $\tilde{n} \circ A=n$, hence that $G=A^{t} \tilde{G} A$.

Proof of Corollary 1.1 If the geodesic flow of the metric $g^{\prime}$ is topologically conjugated to the geodesic flow of the flat metric $g$, then the polynomial entropy of $g^{\prime}$ is equal to the polynomial entropy of $g$, which is equal to 1 . Theorem 1 implies that $g^{\prime}$ is flat, and Proposition 3.1 then implies that $g$ and $g^{\prime}$ are isometric.

\subsection{The special case of dimension two, the main statement in the Tonelli case}

In this section, we work on the two-dimensional torus $\mathbf{T}=\mathbb{R}^{2} / \mathbb{Z}^{2}$. We first recall from [12] some useful facts on rotation sets of flows on $\mathbf{T}$.

Let us consider a flow on $\mathbf{T}$ and lift it to the flow $\psi^{t}$ on the cover $\mathbb{R}^{2}$ [identified with $\left.H_{1}\left(\mathbf{T}, \mathbb{R}^{2}\right)\right]$. The rotation set of our flow is the set of limits of sequences of the form

$$
\left(\psi^{t_{k}}\left(x_{k}\right)-x_{k}\right) / t_{k}
$$

where $x_{k}$ is a sequence in $\mathbb{R}^{2}$ and $t_{k} \longrightarrow \infty$. This is a compact and convex subset of $\mathbb{R}^{2}=H_{1}(\mathbf{T}, \mathbb{R})$ which is not empty. If the flow is generated by a vectorfield $v$, then its rotation set is also the set of the rotation numbers $\int \nu d \mu$ of all invariant probability measures $\mu$. The rotation set of a flow on the two torus $\mathbf{T}$ is a compact interval contained in a straight line through the origin of $\mathbb{R}^{2}=H_{1}(\mathbf{T}, \mathbb{R})$. Moreover, see [12]:

- If the straight line has rational direction [which means that it contains an element of $\left.H_{1}(\mathbf{T}, \mathbb{Z})\right]$, then the ergodic invariant measures of non-zero rotation number are supported on periodic orbits. The $\alpha$ and $\omega$ limit sets of the flow are made of periodic orbits in this case.

- If the straight line has irrational direction, then there is at most one ergodic invariant measure of non-zero rotation number.

Let us apply these results to the Aubry set $\mathcal{A}^{*}(c)$ at a point $c$ which is not a minimum of the function $\alpha$. Then, the rotation set $\partial \alpha(c)$ does not contain zero, and it is contained in the rotation set of the vectorfield $\chi(q)=\partial_{p} H(q, c+d w(q))$ on $\mathbf{T}$. We conclude that $\partial \alpha(c)$ is a compact interval of a ray $\rho(c) \in S H_{1}(\mathbf{T}, \mathbb{R})$, where

$$
\left.\left.S H_{1}(\mathbf{T}, \mathbb{R}):=\left(H_{1}(\mathbf{T}, \mathbb{R})-\{0\}\right) /\right] 0, \infty\right) \approx S^{1}
$$

is the set of open half lines of $H^{1}(\mathbf{T}, \mathbb{R})$ starting at the origin. We say that a ray has rational direction if it contains a point of $H_{1}(\mathbf{T}, \mathbb{Z})$, and that it has irrational direction otherwise. Recall that $c$-minimizing measures are in bijection with $\chi$-invariant probability measures on $\mathcal{A}(c)$.

- If $\rho(c)$ has rational direction, then the ergodic $c$-minimizing measures are supported on periodic orbits. Moreover, the $\alpha$ and $\omega$ limits of each orbit of $\mathcal{A}^{*}(c)$ are periodic orbits supporting $c$-minimizing measures.

- If $\rho(c)$ has irrational direction, then there exists a unique $c$-minimizing measure, and the rotation set $\partial \alpha(c)$ is a point.

In all cases, each half orbit of $\mathcal{A}(c)$ has a single rotation number which is contained in $\rho(c)$. However, in the case of a rational direction, it is possible that the positive half-orbit and the negative half-orbit of a given point have different rotation numbers both contained in $\rho(c)$.

Let us explain a bit more how different rotation numbers can appear in the rational case. In this case, the periodic orbits of $\mathcal{A}(c)$ are oriented embedded closed curve, and they all represent the same homology class $[\rho(c)] \in H_{1}(\mathbf{T}, \mathbb{Z})$ which is the only indivisible integer 
class in the half line $\rho(c)$. The rotation number of the invariant measure supported on such an orbit is then $[\rho(c)] / T$, where $T$ is the minimal period of the orbit. The periodic orbits of $\mathcal{A}(c)$ do not necessarily all have the same period, hence the associated measures do not necessarily have the same rotation number.

For each $e>\min \alpha$, the set

$$
A(e):=\left\{c \in H^{1}(\mathbf{T}, \mathbb{R}): \alpha(c) \leqslant e\right\} \subset H^{1}(\mathbf{T}, \mathbb{R})
$$

is a compact and convex set, whose interior is $\{\alpha<e\}$ and whose boundary is $\alpha^{-1}(e)$. At each boundary point $c \in \alpha^{-1}(e)$, the set $A(e)$ has a single outer normal $\rho(c) \in S H_{1}(\mathbf{T}, \mathbb{R})$. The map $c \longmapsto \rho(c)$ is thus continuous, and the set $\alpha^{-1}(e)$ is a $C^{1}$ curve. Note that the map $\rho: \alpha^{-1}(e) \longrightarrow S H_{1}(\mathbf{T}, \mathbb{R})$ is continuous and onto, and that it preserves the order. It is however not necessarily one to one. For each $c \in \alpha^{-1}(e)$, we consider the face $F(c) \subset \alpha^{-1}(e)$ defined as the set of cohomologies $c^{\prime}$ such that $\rho\left(c^{\prime}\right)=\rho(c)$ and $\alpha\left(c^{\prime}\right)=e$. The face $F(c)$ is a compact segment containing $c$. It is also the set of points $c^{\prime} \in A(e)$ such that $\left(c^{\prime}-c\right) \cdot \rho(c)=0$. The following is well known, see [2,23,25], but since we give the statement in a way which is not obviously equivalent to those of these papers, we will provide a proof in Sect. 3.4. We recall that the Mather set $\mathcal{M}^{*}(c)$ is the union of the supports of $c$-minimizing measures, and that the projected Mather set $\mathcal{M}(c) \subset \mathbf{T}$ is its projection.

Proposition 3.2 Each $c \in \alpha^{-1}(e)$ is in one (and only one) of the following three cases:

1. $\rho(c)$ has irrational direction and $F(c)=\{c\}$.

2. $\rho(c)$ has rational direction, $\mathcal{M}(c)=\boldsymbol{T}$, and $F(c)=\{c\}$

3. $\rho(c)$ has rational direction, $\mathcal{M}(c) \neq \boldsymbol{T}$ and $F(c)$ is a non-trivial segment $\left[c^{-}, c^{+}\right]$. The sets $\mathcal{A}^{*}\left(c^{-}\right)$and $\mathcal{A}^{*}\left(c^{+}\right)$contain non-periodic orbits (which are heteroclinics).

If, for a given value $e>\min \alpha$ of the energy, case 3 does not occur for any $c \in \alpha^{-1}(e)$, then the map $c \longmapsto \rho(c)$ is a homeomorphism from $\alpha^{-1}(e)$ to $S H_{1}(\mathbf{T}, \mathbb{R})$. The energy level $\{H=e\}$ is then $C^{0}$-integrable, as is proved in ([24], Theorem 3), see also Sect. 3.4:

Proposition 3.3 If, for a given value $e>\min \alpha$ of the energy, case 3 does not occur for any $c \in \alpha^{-1}(e)$, then the Aubry sets $\mathcal{A}^{*}(c), c \in \alpha^{-1}(e)$ are Lipschitz invariant graphs which partition the energy level $\{H=e\}$.

If $H$ is the Hamiltonian associated to a Riemaniann metric, then this implies that the metric is flat, in view of the Theorem of Hopf, see also [15]. As a consequence, Theorem 1 follows from:

Theorem 2 Let $e>\min \alpha$ be a given energy level. If there exists a cohomology $c \in \alpha^{-1}(e)$ in case 3, then the polynomial entropy of the Hamiltonian flow restricted to the energy level $\{H=e\}$ is not less than 2. In other words, if the polynomial entropy of the flow restricted to the energy level $\{H=e\}$ is less than two, the Aubry sets $\mathcal{A}^{*}(c), c \in \alpha^{-1}(e)$ are Lipschitz. invariant graphs which partition the energy level.

We will make use in the proof of two important properties of the Aubry sets:

Property 3.1 The set-valed map $c \longmapsto \mathcal{A}^{*}(c)$ is outer semi-continuous. It means that each open set $U \subset T^{*} \boldsymbol{T}$ containing $\mathcal{A}^{*}(c)$, also contains $\mathcal{A}^{*}\left(c^{\prime}\right)$ for $c^{\prime}$ close to $c$.

We recall the definition of the vectorfield $\chi(q):=\partial_{p} H(q, c+d w(q))$ on $\mathbf{T}$. 
Property 3.2 For each $c \in \alpha^{-1}(e)$, there exists a global curve of section of $\mathcal{A}(c)$. More precisely, there exists a cooriented $C^{1}$ embedded circle $\Sigma \subset \boldsymbol{T}$ such that $\chi(q)$ is transverse to $\Sigma$ on $\mathcal{A}(c)$ and respects the coorientation. Moreover, each half orbit of $\mathcal{A}(c)$ intersects $\Sigma$. The flow of $\mathcal{A}(c)$ thus induces a homeomorphism $\psi$ of $\Sigma \cap \mathcal{A}(c)$ which preserves the cyclic order of $\Sigma$.

Proof of Property 3.1 It is proved in [5] using the content of [10].

Proof of Property 3.2 Let us consider a cohomology $c_{0}$ such that $\alpha\left(c_{0}\right)<e$ and such that $c-c_{0} \in H^{1}(\mathbf{T}, \mathbb{Q})$. Since $\rho(c)$ is the outer normal to $\partial A(e)$ at $c$ and $c_{0}$ belongs to the interior of $A(e)$, we have

$$
\rho(c) \cdot\left(c-c_{0}\right)>0 .
$$

We consider a $c$-critical subsolution $w$ of class $C^{1,1}$ and strict outside the Aubry set. We also consider a $c_{0}$-critical subsolution $u_{0}$. Let $l \in \mathbb{N}$ be such that $l\left(c-c_{0}\right) \in H^{1}(\mathbf{T}, \mathbb{Z})$. Let us consider the $C^{1,1}$ function $\hat{\Theta}$ on $\mathbb{R}^{2}$ defined by

$$
q \longmapsto \hat{\Theta}(q)=l\left(c-c_{0}\right) q+l w(q)-l u_{0}(q) .
$$

The function $\tilde{\Theta}=\hat{\Theta} \bmod 1: \mathbb{R}^{2} \longrightarrow \mathbb{T}=\mathbb{R} / \mathbb{Z}$ is $\mathbb{Z}^{2}$-periodic, hence it gives rise to a function $\Theta: \mathbf{T} \longrightarrow \mathbb{T}$ such that $d \Theta=l\left(c-c_{0}\right)+l\left(d w-d u_{0}\right)$. For each point $q$ such that $H(q, c+d w(q))=e$, we have

$$
\begin{aligned}
e-l^{-1} d \Theta(q) \cdot \chi(q)= & H(q, c+d w(q))-\partial_{p} H(q, c+d w(q)) \\
& \cdot\left(c_{0}+d u_{0}(q)-c-d w(q)\right) \\
\leqslant & H\left(q, c_{0}+d u_{0}(q)\right) \leqslant \alpha\left(c_{0}\right)<e
\end{aligned}
$$

hence

$$
d \Theta(q) \cdot \chi(q)>0 \text {. }
$$

Let us consider a regular value $\theta$ of $\Theta$. Such a value exists by fine versions of Sard's Theorem (see [3]) since $\Theta$ is $C^{1,1}$. The preimage $\Theta^{-1}(\theta)$ is a 1-dimensional cooriented submanifold of $\mathbf{T}$. It can be seen as an intersection cocycle of cohomology $l\left(c-c_{0}\right)$. It is a finite union of embedded cooriented circles $\Sigma_{i}$ each of which is a cocycle of cohomology $\sigma_{i}$, with $\sum \sigma_{i}=l\left(c-c_{0}\right)$. Since $\rho(c) \cdot l\left(c-c_{0}\right)>0$, there exists $j$ such that $\rho(c) \cdot \sigma_{j}>0$. We denote by $\Sigma$ the cooriented circle $\Sigma_{j}$. Since $d \Theta(q) \cdot \chi(q)>0$, the orbits of $\mathcal{A}(c)$ are transverse to $\Sigma$, and intersect it according to the coorientation.

Finally, each half orbit of $\mathcal{A}(c)$ has a rotation number contained in $\rho(c)$. We have seen that $\sigma \cdot \rho(c)>0$, where $\sigma$ is the cohomology of the intersection cocycle associated to $\Sigma$. Each half orbit of $\mathcal{A}(c)$ thus intersects $\Sigma$. As a consequence, the flow of $\mathcal{A}(c)$ generates a Poincaré map

$$
\psi: \mathcal{A}(c) \cap \Sigma \longrightarrow \mathcal{A}(c) \cap \Sigma
$$

which is a bi-Lipschitz homeomorphism preserving the cyclic order on the circle $\Sigma$. This implies that $\psi$ can be extended to a homeomorphism of $\Sigma$ preserving the cyclic order.

\subsection{Faces of the balls of $\alpha$ on the two torus}

We take $d=2$ and fix an energy level $e>\min \alpha$. We study the affine parts of the ball $\alpha^{-1}(e)$ and prove Propositions 3.2 and 3.3. The following is a variant of a Lemma of Massart [23]: 
Lemma 3.1 Assume that $d=2$, that $e>\min \alpha$, and that the segment $\left[c_{0}, c_{1}\right]$ is contained in $\alpha^{-1}(e)$. Then the Mather set $\mathcal{M}^{*}(c)$ does not change when $c$ varies in $\left[c_{0}, c_{1}\right]$.

Proof We have $\left(c_{1}-c_{0}\right) \cdot \rho(c)=0$ for each $c \in\left[c_{0}, c_{1}\right]$. Let $c$ be a point in $\left[c_{0}, c_{1}\right]$ and let $\mu^{*}$ be an ergodic $c$-minimizing measure. Such a measure has a rotation number $h\left(\mu^{*}\right)=s \rho(c), s>0$. For each $c^{\prime}$, we have

$$
\alpha\left(c^{\prime}\right) \geqslant \int_{T^{*} \mathbf{T}}\left(c^{\prime}-p\right) \cdot \partial_{p} H(q, p)+H(q, p) d \mu^{*}(q, p)=\alpha(c)+\left(c^{\prime}-c\right) \cdot h\left(\mu^{*}\right) .
$$

If $c^{\prime} \in\left[c_{0}, c_{1}\right]$, then the inequality $\alpha\left(c^{\prime}\right) \geqslant \alpha(c)+\left(c^{\prime}-c\right) \cdot h\left(\mu^{*}\right)$ is an equality, hence $\mu^{*}$ is a $c^{\prime}$-minimizing measure. This implies that $\mathcal{M}^{*}\left(c^{\prime}\right) \subset \mathcal{M}^{*}(c)$ for each $c^{\prime} \in\left[c_{0}, c_{1}\right]$. By symetry, we conclude that $\mathcal{M}^{*}\left(c^{\prime}\right)=\mathcal{M}^{*}(c)$ for $c^{\prime} \in\left[c_{0}, c_{1}\right]$.

The following Lemma also comes from Massart [23]:

Lemma 3.2 Assume that $e>\min \alpha$, and that the segment $\left[c_{0}, c_{1}\right]$ is contained in $\alpha^{-1}(e)$. Then the Aubry set $\mathcal{A}^{*}(c)$ does not change when $c$ varies in $] c_{0}, c_{1}[$. Moreover, we have the inclusion $\mathcal{A}^{*}(c) \subset \mathcal{A}^{*}\left(c_{0}\right) \cap \mathcal{A}^{*}\left(c_{1}\right)$ for each $\left.c \in\right] c_{0}, c_{1}[$.

We will see that, unlike the Mather set, the Aubry set can be bigger at the boundary points.

Proof Let us consider a point $\left.c=a c_{0}+(1-a) c_{1}, a \in\right] 0,1\left[\right.$. Let $w_{i}, i \in\{0,1\}$ be a $c_{i}$-critical subsolution strict outside the Aubry set. Then, $w_{c}:=a w_{0}+(1-a) w_{1}$ is a $c$ critical subsolution. Using the strict convexity of $H$ in $p$, we observe that the strict inequality $H\left(q, c+s w_{c}(q)\right)<e$ holds outside of the set where $H\left(q, c_{0}+d w_{0}(q)\right)=e$ and $H\left(q, c_{1}+\right.$ $\left.d w_{1}(q)\right)=e$ and $c_{0}+d w_{0}=c_{1}+d w_{1}$. We conclude that the Aubry set $\mathcal{A}(c)$ is contained in $\mathcal{A}\left(c_{0}\right) \cap \mathcal{A}\left(c_{1}\right)$, and that $c_{0}+d w_{0}=c_{1}+d w_{1}=c+d w_{c}$ on $\mathcal{A}(c)$. As a consequence, $\mathcal{A}^{*}(c) \subset \mathcal{A}^{*}\left(c_{0}\right) \cap \mathcal{A}^{*}\left(c_{1}\right)$. If $c$ and $c^{\prime}$ are two points in $] c_{0}, c_{1}$ [, assuming for example that $c \in] c_{0}, c^{\prime}$, we conclude that $\mathcal{A}^{*}(c) \subset \mathcal{A}\left(c^{\prime}\right)$. Similarly, we have $\left.c^{\prime} \in\right] c, c_{1}$ [, and we obtain the converse inclusion, hence $\mathcal{A}^{*}(c)=\mathcal{A}^{*}\left(c^{\prime}\right)$.

We recall that $F(c)$ is defined as the largest segment of $\alpha^{-1}(e)$ containing $c$.

Corollary 3.1 If $\mathcal{M}(c)=\boldsymbol{T}$, then $F(c)=c$.

Proof If $\mathcal{M}(c)=\mathbf{T}$, then there exist one and only one $c$-critical subolution $w$, and $\mathcal{M}^{*}(c)$ is the graph of $c+d w$. Assume now that there exists $c^{\prime}$ such that $\mathcal{M}^{*}\left(c^{\prime}\right)=\mathcal{M}^{*}(c)$. Then $\mathcal{M}\left(c^{\prime}\right)=\mathbf{T}$, hence there exists a unique $c^{\prime}$-critical subsolution $w^{\prime}$, and $\mathcal{M}^{*}\left(c^{\prime}\right)$ is the graph of $c^{\prime}+d w^{\prime}$. We thus have $c+d w=c^{\prime}+d w^{\prime}$ at each point, which implies that $c=c^{\prime}$. In view of Lemma 3.1, this implies that $F(c)=c$.

The following was first proved by Bangert, see [2]:

Corollary 3.2 If $\rho(c)$ has an irrational direction, then $F(c)=c$.

Proof As above, let us consider a cohomology $c$ satisfying the hypothesis of the Corollary, and a cohomology $c^{\prime}$ such that $\left[c, c^{\prime}\right] \in \alpha^{-1}(e)$, hence $\mathcal{M}^{*}\left(c^{\prime}\right)=\mathcal{M}^{*}(c)$, by Lemma 3.1. We will prove that $c^{\prime}=c$, which implies the Corollary. Note that $\left(c^{\prime}-c\right) \cdot \rho(c)=0$, so that it is enough to prove that $\left(c^{\prime}-c\right) \cdot[\Sigma]=0$, where $[\Sigma] \in H_{1}(\mathbf{T}, \mathbb{Z})$ is the homology of the section $\Sigma$ given by Property 3.2 (equipped with an orientation). Let $w$ and $w^{\prime}$ be $c$ and $c^{\prime}$-critical subsolutions. We consider the closed Lipschitz form $\eta=c^{\prime}-c+d w^{\prime}-d w$, whose cohomology is $c^{\prime}-c$ and prove that $\int_{\Sigma} \eta=0$. 
Since $\mathcal{M}^{*}\left(c^{\prime}\right)=\mathcal{M}^{*}(c)$, we have $\eta=0$ on $\mathcal{M}(c)$. It is thus enough to prove that $\int_{I} \eta=0$ for each connected component $I$ of the complement of $\mathcal{M}(c) \cap \Sigma$ in $\Sigma$. We first observe that

$$
\int_{I} \eta=\int_{\psi(I)} \eta
$$

where $\psi$ is a homeomorphism of $\Sigma$ extending the return map of $\Sigma \cap \mathcal{A}(c)$. To prove this equality, we integrate $\eta$ on the contractible closed curve made of the interval $I=] q^{-}, q^{+}[$, followed by the orbit of $q^{+}$until its return $\psi\left(q^{+}\right)$, followed by the interval $-\psi(I)=$ ] $\psi\left(q^{+}\right), \psi\left(q^{-}\right)$[ followed by the piece of orbit of $q^{-}$in negative time direction from $\psi\left(q^{-}\right)$ to $q^{+}$.

Since the intervals $\psi^{k}(I)$ are two by two disjoint in $\Sigma$, their lengh is converging to zero. Since the form $\eta$ is bounded, this implies that $\int_{\psi^{k}(I)} \eta \longrightarrow 0$, hence that $\int_{I} \eta=0$.

In view of these corollaries there are three cases:

- $\rho(c)$ has irrational direction and $F(c)=\{c\}$ (Corollary 3.2).

- $\rho(c)$ has rational direction, $\mathcal{M}(c)=\mathbf{T}$, and $F(c)=\{c\}$ (Corollary 3.1).

- $\rho(c)$ has rational direction and $\mathcal{M}(c) \neq \mathbf{T}$.

Let us study more precisely the last case. We denote by $\left[c^{-}, c^{+}\right]$the face $F(c)$. We assume for definiteness that $c$ is an interior point of this face, which means that either $c \in] c^{-}, c^{+}[$ or $c^{-}=c=c^{+}$.

We consider the cooriented section $\Sigma$ given by Property 3.2. We orient $\Sigma$ in such a way that $\left(c^{+}-c^{-}\right) \cdot[\Sigma] \geqslant 0$, where $[\Sigma]$ is the homology of $\Sigma$ (hence $\left(c^{+}-c^{-}\right) \cdot[\Sigma]>0$ if $c^{+} \neq c^{-}$, since $[\Sigma]$ is not proportional to $\rho(c))$. The return map $\psi$ from $\mathcal{M}(c) \cap \Sigma$ to istelf is periodic, its minimal period is $\tau:=\sigma \cdot[\rho(c)]$. The complement of $\mathcal{M}(c)$ in $\mathbf{T}$ is a disjoint union of topological open annuli. Each of these annuli $U$ intersects $\Sigma$ in $\tau$ disjoint open intervals, that we orient according to the orientation of $\Sigma$. Each orbit of $\mathcal{A}(c)-\mathcal{M}(c)$ is contained in such an annulus $U$, is $\alpha$-asymptotic to one of its boundaries, and is $\omega$-asymptotic to its other boundary. We say that such an orbit is positive if it crosses the annulus $U$ according to the orientation of $\Sigma$, and that it is negative if it crosses in the other direction. In other words, the heteroclinic orbit is positive if the sequence of its successive intersections with the interval $I$ is increasing. The following implies Proposition 3.2:

Proposition 3.4 If $c \in \alpha^{-1}(e)$ is such that $\rho(c)$ is rational and $\mathcal{M}(c) \neq \boldsymbol{T}$, then

- $c^{-} \neq c^{+}$

- The Aubry set $\mathcal{A}\left(c^{+}\right)$contains positive heteroclinics in each connected component of $\boldsymbol{T}-\mathcal{M}(c)$ and no other orbit except those of $\mathcal{M}(c)$.

- The Aubry set $\mathcal{A}\left(c^{-}\right)$contains negative heteroclinics in each connected component of $\boldsymbol{T}-\mathcal{M}(c)$ and no other orbit except those of $\mathcal{M}(c)$.

- Finally, $\mathcal{A}(c)=\mathcal{M}(c)$ for each $c$ in $] c^{-}, c^{+}[$.

Proof The statements concerning $\mathcal{A}\left(c^{+}\right)$and $\mathcal{A}\left(c^{-}\right)$imply that $c^{+} \neq c^{-}$. Moreover, they imply that $\mathcal{A}\left(c^{+}\right) \cap \mathcal{A}\left(c^{-}\right)=\mathcal{M}(c)$, hence that $\mathcal{A}(c)=\mathcal{M}(c)$ for each $c$ in $] c^{-}, c^{+}$, by Lemma 3.2.

We will now prove the statement concerning $\mathcal{A}\left(c^{+}\right)$, the other one being similar. We fix a connected component $U$ of $\mathbf{T}-\mathcal{M}(c)$, and a connected component $I$ of $U \cap \Sigma$. Let $\rho_{m}$ be the direction of $m[\rho]+[\Sigma]$, and let $c_{m} \in \alpha^{-1}(e)$ be such that $\rho\left(c_{m}\right)=\rho_{m}$. Note that $c_{m} \longrightarrow c^{+}$.

The annulus $U$ contains an oriented closed curve $K$ of homology $[\rho]$. The Aubry set $\mathcal{A}\left(c_{m}\right)$ contains a periodic orbit of homology positively proportional to $m[\rho]+[\Sigma]$, hence 
it intersects $K$. In view of the semi-continuity of the Aubry set, see Property 3.1, we deduce that $\mathcal{A}\left(c^{+}\right)$intersects $K$. As a consequence, the set $\mathcal{A}\left(c^{+}\right)$does contain heteroclinic orbits in $U$. Moreover, there exists $m_{0} \in \mathbb{N}$ and a compact subinterval $J \subset I$ which contains a point in each orbit of $\mathcal{M}\left(c_{m}\right)$ for $m \geqslant m_{0}$.

Let us now consider the return map $\psi^{\tau}$ of $I \cap \mathcal{A}(c)$. Either we have $\psi^{\tau}(x)>x$ for each $x \in I \cap \mathcal{A}(c)$, and the orbits of $\mathcal{A}(c) \cap U$ are positive heteroclinics, or we have $\psi^{\tau}(x)<x$ for each $x \in I \cap \mathcal{A}(c)$, and the orbits of $\mathcal{A}(c) \cap U$ are negative heteroclinics.

In view of the semi-continuity of the Aubry set, $\Sigma$ is also a cooriented transverse section for $\mathcal{A}\left(c_{m}\right)$ for $m \geqslant m_{0}$, provided $m_{0}$ is large enough. Denoting by $\psi_{c_{m}}$ the corresponding section map, we have $\psi_{c_{m}}^{\tau}(J) \subset I$ for $m \geqslant m_{0}$ provided $m_{0}$ is large enough. Then, there exists a point $x_{m} \in J \cap \mathcal{M}\left(c_{m}\right) \subset J \cap \mathcal{A}\left(c_{m}\right)$, and $x_{m}<\psi_{c_{m}}^{\tau}\left(x_{m}\right)$.

At the limit, using the semi-continuity of the Aubry set, we find a point $x \in J \cap \mathcal{A}\left(c^{+}\right)$ such that $\psi^{\tau}(x) \geqslant x$, hence $\psi^{\tau}(x)>x$. We conclude that the heteroclinics are positive.

For the convenience of the reader, and because our statement is not exactly the one of [24], Theorem 3, we now prove Proposition 3.3, following [24]:

We consider an energy level $e>\min \alpha$ such that the curve $\alpha^{-1}(e)$ does not contain any non-trivial segment, which is equivalent to saying that $\mathcal{M}(c)=\mathbf{T}$ for each $c$ such that $\rho(c)$ is rational. Note then that the map $\rho: \alpha^{-1}(e) \longrightarrow S H_{1}(\mathbf{T}, \mathbb{R})$ is continuous and bijective, hence it is a homeomorphism. Since the set $S H_{1}(\mathbf{T}, \mathbb{Z})$ of rational directions is dense in $S H_{1}(\mathbf{T}, \mathbb{R})$, its preimage $\rho^{-1}\left(S H_{1}(\mathbf{T}, \mathbb{Z})\right)$ is dense in $\alpha^{-1}(e)$. For each point $c$ in this set, we have $\mathcal{A}(c)=\mathbf{T}$. In view of the semi-continuity of the Aubry set, we deduce that $\mathcal{A}(c)=\mathbf{T}$ for each $c \in \alpha^{-1}(e)$. As a consequence, there exists a unique (up to the addition of a constant) $c$-critical subsolution $w_{c}$, which is actually a solution, and the Aubry set $\mathcal{A}^{*}(c)$ is the graph of $c+d w_{c}$. Moreover, the functions $d w_{c}, c \in \alpha^{-1}(e)$ are equi-Lipschitz. The semicontinuity of the Aubry set $\mathcal{A}^{*}$ implies that the map $c \longmapsto c+d w_{c}(q)$ is continuous for each $q \in \mathbf{T}$.

The orbits of $\mathcal{A}^{*}(c)$ all have a forward rotation number in $\rho(c)$. For $c^{\prime} \neq c$, the orbits of $\mathcal{A}^{*}\left(c^{\prime}\right)$ all have a forward rotation number in $\rho\left(c^{\prime}\right)$, and, since $\rho\left(c^{\prime}\right) \neq \rho(c)$, the sets $\mathcal{A}^{*}(c)$ and $\mathcal{A}^{*}\left(c^{\prime}\right)$ are disjoint. As a consequence, for each $q \in \mathbf{T}$, the map $c \longmapsto c+d w_{c}(q)$ is one to one on $\alpha^{-1}(e)$, hence it has degree \pm 1 as a circle map into $\left\{p \in T_{q} \mathbf{T}: H(q, p)=e\right\}$. It is thus onto, which implies that the Aubry sets fill the energy level.

\section{Lower bound for the polynomial entropy}

We prove Theorem 2. We consider an energy level $e>\min \alpha$, assume that the ball $\alpha^{-1}(e)$ contains a non-trivial face $\left[c^{-}, c^{+}\right]$, and prove that the entropy of the Hamiltonian flow on the energy level $H^{-1}(e)$ is at least two. The proof have similarities with the ones of [19,21,22]. We work with the section $\Sigma$ of $\mathcal{A}\left(c^{+}\right)$given in Property 3.2. We fix a parameterisation $\mathbb{R} / \mathbb{Z} \longrightarrow \Sigma$, and put on $\Sigma$ the distance such that this parameterisation is isometric. This distance is Lipschitz equivalent to the restriction of the distance on $\mathbf{T}$.

The direction $\rho(c) \in S H_{1}(\mathbf{T}, \mathbb{Z})$ is independant of $c \in\left[c^{-}, c^{+}\right]$, and it is rational, we denote it by $\rho$ in the sequel, and by $[\rho] \in H_{1}(\mathbf{T}, \mathbb{Z})$ the associated indivisible integer point. As above, we consider, for $m \in \mathbb{N}$, the direction $\rho_{m}$ of $m[\rho]+[\Sigma]$ and a cohomology $c_{m} \in \alpha^{-1}(e)$ such that $\rho\left(c_{m}\right)=\rho_{m}$. Let us decide for definiteness that $c_{m} \longrightarrow c^{+}$(otherwise we exchange the names of $c^{+}$and $c^{-}$). We fix $m_{0}$ large enough so that $\Sigma$ is a cooriented transverse section of $\mathcal{A}\left(c_{m}\right)$ for each $m \geqslant m_{0}$ (such a value of $m_{0}$ exists in view of the semi-continuity of the Aubry set) and denote by $\psi_{c_{m}}$ the corresponding return map of $\mathcal{A}\left(c_{m}\right) \cap \Sigma$. The orbits of $\mathcal{M}(c)$ give rise to periodic orbits of $\psi$, and the minimal period of these orbits is $\tau:=\sigma \cdot[\rho]$, 
where $\sigma$ is the cohomology of the intersection cocycle associated to $\Sigma$. The integral class $m[\rho]+[\Sigma]$ is not necessarily indivisible in $H_{1}(\mathbf{T}, \mathbb{Z})$, hence the minimal period for the return map $\psi_{c_{m}}$ of the points of $\Sigma \cap \mathcal{M}\left(c_{m}\right)$ may be smaller than $m \tau$. However, because $m[\rho]+[\Sigma]$ is indivisible in the group generated by $[\Sigma]$ and $[\rho]$, we have:

Lemma 4.1 Each point of $\mathcal{M}\left(c_{m}\right) \cap \Sigma$ is periodic for the map $\psi_{c_{m}}^{\tau}$, and has a minimal period equal to $\mathrm{m}$.

The following proof might appear unnecessarily complicated. Things can also be under-

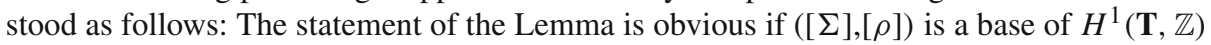
(since $m[\rho]+[\Sigma]$ is then indivisible in $H^{1}(\mathbf{T}, \mathbb{Z})$ ) and we can reduce the situation to this simple case by taking a finite covering, which does not change the value of the polynomial entropy.

Proof Let us denote by $G$ the subgroup of $H_{1}(\mathbf{T}, \mathbb{Z})$ generated by $[\Sigma]$ and $[\rho]$. Let $\chi: \mathbf{T} \longrightarrow$ $\mathbf{T}$ be a covering such that $\chi_{*}\left(H_{1}(\mathbf{T}, \mathbb{Z})\right)=G$. This coverging has $\tau$ sheets. The preimage of $\Sigma$ by this coverging has $\tau$ connected components, and we denote by $\tilde{\Sigma}$ one of them. We have

$$
[\tilde{\Sigma}]=\chi_{*}^{-1}([\Sigma]) \subset H_{1}(\mathbf{T}, \mathbb{Z}),
$$

and the cohomology of the intersection cocycle associated to $\tilde{\Sigma}$ is $\tilde{\sigma}:=\chi^{*}(\sigma) / \tau$. The preimage of each orbit of $\mathcal{M}(\rho)$ has $\tau$ connected components, each of which is a closed curve of homology $[\tilde{\rho}]:=\chi_{*}^{-1}([\rho])$. Since $\left[\rho_{m}\right]$ does not necessarily belong to $G$, the preimage of closed orbits in $\mathcal{M}\left(c_{m}\right)$ may have less than $\tau$ connected components. Each of these components have a homology in $H_{1}(\mathbf{T}, \mathbb{Z})$ which is indivisible and positively proportional to $\left[\tilde{\rho}_{m}\right]:=m[\tilde{\Sigma}]+[\tilde{\rho}]$. Since $m[\rho]+[\Sigma]$ is indivisible in $G,\left[\tilde{\rho}_{m}\right]$ is indivisible in $H_{1}(\mathbf{T}, \mathbb{Z})$, hence it is equal to the homology of the connected components of the preimages of orbits of $\mathcal{M}\left(c_{m}\right)$. Note that $\chi_{*}\left(\left[\tilde{\rho}_{m}\right]\right)=m[\rho]+[\Sigma]$ is not necessarily equal to $\left[\rho_{m}\right]$.

The minimal $\psi_{c_{m}}^{\tau}$-period of orbits of $\mathcal{M}\left(c_{m}\right) \cap \Sigma$ is equal to the intersection number $\tilde{\sigma} \cdot\left[\tilde{\rho}_{m}\right]=(\sigma / \tau) \cdot(m[\rho]+[\Sigma])=m$.

As in the proof of Proposition 3.4, we consider a compact subinterval $J \subset I$ such that each orbit of $\mathcal{A}\left(c_{m}\right)$ contains a point of $J$ for $m \geqslant m_{0}$ (we may have to increase $m_{0}$ ). We can chose $\epsilon_{0}>0$ small enough and $m_{0}$ large enough to have, for all $m \geqslant m_{0}$,

$$
d\left(q, \psi_{c_{m}}^{ \pm \tau}(q)\right) \geqslant 2 \epsilon_{0}
$$

for all $q \in \mathcal{A}\left(c_{m}\right) \cap I$ such that $d(q, J) \leqslant \epsilon_{0}$. Each point of $J \cap \mathcal{M}\left(c_{m}\right), m \geqslant m_{0}$ is at distance at least $2 \epsilon_{0}$ from all the other points of its $\psi^{\tau}$-orbit. We deduce:

Lemma 4.2 For $q \in J \cap \mathcal{M}\left(c_{m}\right), m \geqslant m_{0}$, the orbit

$$
O_{\psi^{\tau}}(q):=\left\{q, \psi_{c_{m}}^{\tau}(q), \psi_{c_{m}}^{2 \tau}(q), \ldots, \psi_{c_{m}}^{(m-1) \tau}(q)\right\}
$$

is $\left(\epsilon_{0}, m\right)$-separated by $\psi^{\tau}$, hence $\left(2 \epsilon_{0}, \tau m\right)$-separated by $\psi$.

Proof Let $\theta$ and $\theta^{\prime}$ be two points of this orbit. There exists $l \in\{0,1, \ldots, m-1\}$ such that $\psi^{l \tau}(\theta) \in J$. Then $\psi^{l \tau}\left(\theta^{\prime}\right)$ is another point of the same orbit, hence $d\left(\psi^{l \tau}(\theta), \psi^{l \tau}\left(\theta^{\prime}\right)\right) \geqslant 2 \epsilon_{0}$. 
We denote by $\Sigma^{*}$ the set of points of the energy surface $H^{-1}(e)$ which project on $\Sigma$, we endow it with a distance which satisfies $d\left((q, p),\left(q^{\prime}, p^{\prime}\right)\right) \geqslant d\left(q, q^{\prime}\right)$. We consider the compact invariant set of the Hamiltonian flow (on the energy level) defined by

$$
A:=\mathcal{A}^{*}\left(c^{+}\right) \cup \bigcup_{m \geqslant m_{0}} \mathcal{A}^{*}\left(c_{m}\right) .
$$

The surface $\Sigma^{*}$ is a transverse section of the flow on this invariant set, as required in Proposition 2.1. We denote by $\Psi$ the corresponding return map of $A \cap \Sigma^{*}$. The restriction of $\Psi$ to $\mathcal{A}^{*}\left(c_{m}\right) \cap \Sigma^{*}$ is conjugated to $\psi_{c_{m}}$ by the projection. In view of Proposition 2.1 , it is enough to bound from below the polynomial entropy of $\Psi$ on $A \cap \Sigma^{*}$. We exhibit a sufficiently large separated set using the orbits

$$
O_{\Psi^{\tau}}(x):=\left\{x, \Psi^{\tau}(x), \ldots, \Psi^{(k-1) \tau}(x)\right\} .
$$

Lemma 4.3 Let us chose, for each $m \geqslant m_{0}$, an element $x_{m}$ of $\mathcal{M}^{*}\left(c_{m}\right) \cap J^{*}$. The set

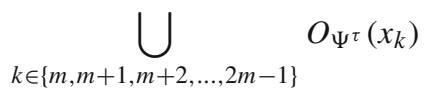

is $\left(\epsilon_{0}, 4 m\right)$-separated by $\Psi^{\tau}$, hence $\left(\epsilon_{0}, 4 m \tau\right)$-separated by $\Psi$ (for $\left.m \geqslant m_{0}\right)$.

Since the cardinal of this union is more than $\mathrm{m}^{2}$, we conclude that the polynomial entropy of $\Psi$ is at least two. By Proposition 2.1 the polynomial entropy of the Hamiltoinan flow on the energy surface is at least two.

Proof Let $x=(q, p) \in O_{\Psi^{\tau}}\left(x_{k}\right)$ and $y=(\theta, \eta) \in O_{\Psi^{\tau}}\left(x_{l}\right)$ be two different points in this union.

If $k=l$, the points $x$ and $y$ belong to the same orbit $O_{\Psi^{\tau}}\left(x_{k}\right)$. They are $\left(\epsilon_{0}, m\right)$-separated by $\Psi^{\tau}$ in view of Lemma 4.2.

Otherwise, we assume for definiteness that $m \leqslant k<l<2 m$. There exists an integer $s \in\{0,1, \ldots, k-1\}$ such that $\psi_{c_{k}}^{s \tau}(q) \in J$.

If $d\left(\psi_{c_{k}}^{s \tau}(q), \psi_{c_{l}}^{s \tau}(q)\right) \geqslant \epsilon_{0}$, then $d\left(\Psi^{s \tau}(x), \Psi^{s \tau}(y)\right) \geqslant \epsilon_{0}$ hence $x$ and $y$ are $\left(\epsilon_{0}, k\right)$ separated by $\Psi^{\tau}$.

If $d\left(\psi_{c_{k}}^{s \tau}(q), \psi_{c_{l}}^{s \tau}(\theta)\right) \leqslant \epsilon_{0}$, then $d\left(\psi_{c_{l}}^{s \tau}(\theta), J\right) \leqslant \epsilon_{0}$ hence (3) implies that

$$
d\left(\psi_{c_{l}}^{(k+s) \tau}(\theta), \psi_{c_{l}}^{s \tau}(\theta)\right) \geqslant 2 \epsilon_{0}
$$

so that

$$
d\left(\psi_{c_{l}}^{(k+s) \tau}(\theta), \psi_{c_{k}}^{(k+s) \tau}(q)\right)=d\left(\psi_{c_{l}}^{(k+s) \tau}(\theta), \psi_{c_{k}}^{s \tau}(q)\right) \geqslant \epsilon_{0}
$$

hence

$$
d\left(\Psi^{(k+s) \tau}(y), \Psi^{(k+s) \tau}(x)\right) \geqslant \epsilon_{0} .
$$

As a consequence, the points $x$ and $y$ are $\left(\epsilon_{0}, 4 m\right)$-separated by $\Psi^{\tau}$.

Open Access This article is distributed under the terms of the Creative Commons Attribution 4.0 International License (http://creativecommons.org/licenses/by/4.0/), which permits unrestricted use, distribution, and reproduction in any medium, provided you give appropriate credit to the original author(s) and the source, provide a link to the Creative Commons license, and indicate if changes were made. 


\section{References}

1. Arcostanzo, M., Arnaud, M.-C., Bolle, P., Zavidovique, M.: Tonelli Hamiltonians Without Conjugate Points and $C^{0}$ Integrability. arXiv:1309.6076

2. Bangert, V.: Minimal geodesics. Ergod. Theory Dyn. Syst. 10(2), 263-286 (1990)

3. Bates, S.: Toward a precise smoothness hypothesis in Sard's theorem. Proc. Am. Math. Soc. 117(1), 279-283 (1993)

4. Bernard, P.: Existence of $C^{1,1}$ critical sub-solutions of the Hamilton-Jacobi equation on compact manifolds. Annales scientifiques de l'Ecole normale supérieure 40(3), 445-452 (2007)

5. Bernard, P.: On the Conley decomposition of Mather sets. Revista Matematica Iberoamericana 26(1), 115-132 (2010)

6. Besson, G., Courtois, G., Gallot, S.: Minimal entropy and Mostow's rigidity theorems. Ergod. Theory Dyn. Syst. 16(4), 623-649 (1996)

7. Burago, D., Ivanov, S.: Riemannian tori without conjugate points are flat. Geom. Funct. Anal. 4(3), 259-269 (1994)

8. Contreras, G., Iturriaga, R., Paternain, G.P., Paternain, M.: Lagrangian graphs, minimizing measures and Mañé's critical values. Geom. Funct. Anal. 8(5), 788-809 (1998)

9. Corke, C.B., Kleiner, B.: Conjugacy and rigidity for manifolds with a parallel vector field. J. Diff. Geom. 39, 659-680 (1994)

10. Fathi, A., Rifford, L., Figalli, A.: On the Hausdorff dimension of the Mather quotient. Commun. Pure Appl. Math. 62(4), 445-500 (2009)

11. Fathi, A., Siconolfi, A.: Existence of $C^{1}$ critical sub-solutions of the Hamilton-Jacobi equation. Invent. Math. 155(2), 363-388 (2004)

12. Franks, J., Misiurewicz, M.: Rotation sets of toral flows. Proc. Am. Math. Soc. 109(1), 243-249 (1990)

13. Heber, J.: On the geodesic flow of tori without conjugate points. Math. Z. 216(2), 209-216 (1994)

14. Hopf, E.: Closed surfaces without conjugate points. Proc. Natl. Acad. Sci. U.S.A. 34, 47-51 (1948)

15. Innami, N.: Families of geodesics which distinguish flat tori. Math. J. Okayama Univ. 28(207-217), 1986 (1987)

16. Katok, A.: Four applications of conformal equivalence to geometry and dynamics. Ergod. Theory Dyn. Syst. 8*(Charles Conley Memorial Issue), 139-152 (1988)

17. Labrousse, C.: Polynomial growth of the volume of balls for zero-entropy geodesic systems. Nonlinearity 25(11), 3049-3069 (2012)

18. Labrousse, C.: Flat metrics are strict local minimizers for the polynomial entropy. Regul. Chaotic Dyn. 17(6), 479-491 (2012)

19. Labrousse, C., Marco, J.-P.: Polynomial entropies for Bott nondegenerate Hamiltonian systems. Regul. Chaotic Dyn. 19(3), 374-414 (2014)

20. Mañé, R.: On the minimizing measures of Lagrangian dynamical systems. Nonlinearity 5(3), 623 (1992)

21. Marco, J.-P.: What are the Simplest Smooth Convex Billiard Tables? Preprint ArXiv

22. Marco, J.-P.: Polynomial entropies and integrable Hamiltonian systems. Regul. Chaotic Dyn. 18(6), 623655 (2013)

23. Massart, D.: On Aubry sets and Mather's action functional. Isr. J. Math. 134, 157-171 (2003)

24. Massart, D., Sorrentino, A.: Differentiability of Mather's average action and integrability on closed surfaces. Nonlinearity 24(6), 1777-1793 (2011)

25. Mather, J.N.: Order structure on action minimizing orbits. In: Symplectic Topology and Measure Preserving Dynamical Systems. Contemp. Math. AMS 512, 41-125 (2010)

26. Mather, J.N.: Action minimizing invariant measures for positive definite Lagrangian systems. Math. Z. 207(1), 169-207 (1991)

27. Wilkins, D.: A Course in Riemannian Geometry. Trinity College, Dublin (2005) 